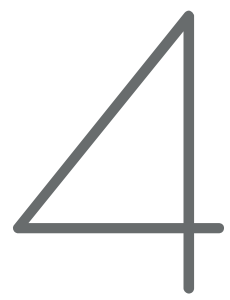

\title{
DATOS SOCIO-ECONÓMICOS Y CULTURALES SOBRE UNA COMUNIDAD ESMERALDEÑA
}

\section{Harald Einzmann}




\section{(1) INTRODUCCIÓN}

La investigación, base de este pequeño estudio, se realizó dentro del programa de investigaciones del Centro Interamericano de Artesanías y Artes Populares, en un período de 4 semanas, en los meses de Noviembre y Diciembre de 1983.

El trabajo se realizó en un ambiente de confianza, gracias a la franqueza natural de los moradores de Same, que facilitó las relaciones amistosas con los investigadores. Así mismo se contó con el apoyo oficial de las autoridades provinciales y locales.

En una reunión inicial, a la que concurrieron la mayoría de los pobladores del recinto, se les habló de los objetivos de nuestra presencia en el lugar; es decir del estudio que se iba a realizar. Se les solicitó su colaboración y la ofrecieron de buen grado.

En el mencionado tiempo, el investigador visitó personalmente a 45 familias, además obtuvo información de 4 familias más, a través de parientes.

Las 41 personas que figuran como informantes principales, se clasifican en 34 hombres y 17 mujeres, de distintas edades.

En la investigación, además de la entrevista, se aplicó un cuestionario (véase apéndice), el mismo que fue modificado y complementado a lo largo del trabajo y de acuerdo con situaciones particulares.

\section{(2) DATOS DEMOGRÁFICOS}

Los integrantes de las 49 familias investigadas comprenden un total de 334 individuos, incluidos los miembros de la familia que aunque no viven en el recinto, son considerados parte de la comunidad; por ejemplo los hijos casados que trabajan fuera del lugar, pero que regresan con frecuencia a él. 
En esta situación se encuentran 42 personas, por lo que el número real de investigados, se reduce a 292 individuos.

Por otra parte, se debe aclarar que no todos los integrantes de la familia mantienen relaciones de parentesco lineal (abuelos, padres e hijos), sino que en algunos casos sólo hay relación de parentesco colateral (cuñados, primos, etc.). De los 292 sujetos, 17 se encuentran en esta situación.

Finalmente, en algunas familias existen individuos, que sin tener ninguna relación de parentesco, "completan" el núcleo familiar. Por ejemplo un hijo adolescente de una familia amiga, o un llamado "veterano"; éste último a veces tiene la función social temporal de "cabeza" del hogar, en ausencia del padre o esposo legítimo.

En cuanto a la edad, el $52 \%$ de las personas investigadas son menores de 20 años y el 69,4 \% son menores de 30 años.

Con respecto a la procedencia, únicamente el 37,27\% de los informantes principales son naturales del lugar (13 hombres y 6 mujeres); es decir que la mayoría inmigraron de otros lugares. De la misma provincia vinieron 12 hombres y 7 mujeres. De la provincia de Manabí proceden 5 hombres y 3 mujeres. Dos hombres llegaron de la provincia de Lo ja y una ancianita es de origen colombiano.

Si clasificamos a los investigados por el sexo, concluimos que el número de varones es mayor que el de mujeres; este resultado concuerda con los datos obtenidos en otro recinto de la costa ecuatoriana (Tonchigüe: 390 hombres y 327 mujeres) y va de acuerdo también con la información proporcionada por INEC según el censo de 1982, con respecto a varias zonas litorales del País.

El fenotipo más frecuente es "el montubio", conocido como el "morenito", con perceptibles variantes por la presencia de sangre negra. También existe un número considerable de representantes de la raza negra pura.. En esta comunidad está ausente el tipo "'serrano" a excepción de los dos lojanos advenedizos. 


\section{(3) UBICACIÓN E INFRAESTRUCTURA}

El recinto de Same se encuentra ubicado a $45 \mathrm{~km}$. al Suroeste de la ciudad de Esmeraldas; a $18 \mathrm{~km}$. al Sur de Atacames. Aproximadamente a $0^{\circ}$ $50^{\prime}$ latitud Norte y $79^{\circ} 55^{\prime}$ longitud Oeste.

Los terrenos ocupados por esta comunidad campesina, comprenden una franja de $5 \mathrm{~km}$. a cada lado de la nueva carretera que une Muisne a Esmeraldas; guardan una distancia aproximada de $2 \mathrm{~km}$. de la playa.

Los terrenos próximos al mar han sido abandonados por los moradores de Same, debido a las mareas altas de los últimos años. Estos terrenos en la actualidad pertenecen a personas extrañas que han construido sus casas vacacionales, o han realizado plantaciones de palmeras. También se han construido dos hoteles.

El centro del caserío está rodeado de tierras bajas y esteros; como en otros recintos, sobresalen la capilla, la escuela y unas pocas tiendas. Hacia el Este se extiende la región montañosa con pequeñas mesetas hasta de 250 metros sobre el nivel del mar y una espesa vegetación tropical.

Según los informes obtenidos, esta comunidad existe desde los años cuarenta y en la actualidad consta de 57 viviendas con alrededor de 328 habitantes.

La denominada "nueva carretera" que une el cantón Muisne a la Capital de la Provincia, facilita la movilización a los moradores de Same, ya sea a las ciudades mencionadas, como también hacia las parroquias de Súa y Atacames, permitiéndoles la comercialización de camarones y langostinos y de los productos agrícolas de la zona.

En este recinto, con excepción de una moto, no existen vehículos particulares; del mismo modo del servicio de energía eléctrica, disfrutan un reducido número de familias, la mayoría prefiere utilizar las lámparas de kérex u otro combustible, debido al elevado costo de la instalación eléctrica (en el momento de la investigación: 6.000 sucres aproximadamente).

Al igual que Tonchigüe, Same tampoco dispone del servicio telefónico, Los servicios de IETEL más cercanos están instalados en Súa y Atacames. En lo que respecta a los servicios de correos, los usuarios tienen 
que dirigirse a las oficinas de la capital de la provincia, aproximadamente una hora en bus. En forma casual algunos choferes de las empresas actúan como carteros.

El primer centro de educación primaria fundado en 1951 fue abandonado en la década pasada, al igual que las demás construcciones que se levantaron en la playa. Posteriormente se construyó otra escuela con el nombre de "FAE" en 1975, pero por su ubicación muy distante de la población, también fue abandonada $\mathrm{y}$, desde 1982 funciona la escuela "Adalberto Ortiz" situada en el centro del caserío.

La parroquia de Tonchigüe a la que pertenece Same, cuenta con una escuela, un colegio y con los servicios de un dispensario médico.

En Same los moradores carecen de los servicios de agua potable, obteniendo este líquido de pozos no muy profundos o directamente del estero. Tampoco existe un sistema de alcantarillado.

Las únicas instalaciones sanitarias son las letrinas que por su ubicación cercana a los pozos, ofrecen un gran peligro de contaminación sobre todo en el invierno con las inundaciones frecuentes.

La población de Same se compone en su mayoría de trabajadores que prestan sus servicios en poblaciones más grandes fuera de su comunidad; unos cuantos agricultores, pescadores y comerciantes.

Las tres pequeñas tiendas mantienen una oferta muy limitada, razón por la cual los moradores prefieren adquirir todos los artículos en poblaciones cercanas. Del mismo modo el único salón de la localidad, atiende solamente los fines de semana.

Los dos hoteles tipo "cabaña" que han sido instalados en la playa, son de propiedad de extranjeros y tienen una selecta clientela de turistas nacionales y extranjeros, sobre todo en determinadas épocas del año; es entonces cuando sus dueños ofrecen trabajo temporal o permanente a los moradores de Same. 


\section{(4) VIVIENDAS: APARIENCIA Y CONSTRUCCIÓN}

Con poquísimas excepciones en el centro poblado, en los demás casos las viviendas están construidas con materiales que les ofrece la naturaleza de la zona: madera de distinta clase, caña guadua y hojas de palmera u otras que sirven para el efecto.

Por lo general, debido a la humedad y a las frecuentes inundaciones, todas las casas tienen una plataforma sobre postes que varían desde un metro, hasta los tres metros de altura. Cuando esta plataforma es alta, el espacio que resulta debajo, es utilizado en el verano como cocina, sala o espacio destinado a la crianza de animales domésticos. En muy pocos casos esta parte de la casa, tiene paredes.

En la parte alta de la casa están los dormitorios, a veces una salita y raras veces también la cocina. Una varanda con la grada conducen a esta parte de la casa. La división se realiza con tablas o tiras de bambú; normalmente las casas no tienen más que dos dormitorios y a veces sólo una cortina los separa.

Los muebles de la sala comprenden una mesa, una o dos bancas y ocasionalmente un estante. En los dormitorios generalmente la ropa está colgada en clavos o cuerdas; las camas son simples armaduras con colchones y a veces una estera colocada en el piso cumple con la misma finalidad.

En el lugar destinado a la cocina, se encuentra el fogón construido de un cajón de tabla o guadua lleno de tierra, con unas varillas de hierro a cierta altura, para colocar en ellas las ollas, en su mayor parte de aluminio.

Como combustible utilizan la leña, ramas u hojas secas; en muy pocos hogares se encuentran las cocinas de gas.

El terreno en el que se asientan las construcciones en algunos casos no es propio, es decir sus ocupantes carecen de títulos de propiedad, pero su ocupación se basa en el llamado "derecho de posesión".

El área normal de los terrenos es de 10 por 20 metros, en donde construyen sus casas y en los espacios sobrantes siembran algunas hortalizas y plantas ornamentales. 
El costo de construcción varía de una vivienda a otra, naturalmente de acuerdo con el material utilizado y las dimensiones de la casa. Según la información proporcionada una casa de bambú y techo de cinc, al tiempo de esta investigación puede costar entre los 10.000 y 30.000 sucres. Este siempre es un dato aproximado, ya que es muy difícil estimar con precisión los costos de la construcción debido a las siguientes razones:

a) La construcción se realiza en el ambiente familiar de colaboración mutua, sólo ocasionalmente se encargan determinados trabajos a un carpintero o persona contratada.

b) Gran parte de los materiales utilizados son obtenidos en terrenos propios de las fincas de los alrededores; en otras ocasiones algo de los materiales se obtiene de las casas viejas semidestruidas por el invierno y las inundaciones.

De esta manera el costo antes mencionado cubre únicamente el valor de los materiales que hay que comprar en los almacenes (cinc, clavos, a veces cemento y algo más).

Para una pequeña casa de hormigón y bloques, en el momento de la investigación, se calculaba que era necesario invertir más de 250.000 sucres sólo en material, sin contar los gastos de mano de obra; por este alto costo, esta clase de construcciones se realiza por etapas y a veces demora algunos años en concluirlas.

En pocos casos los que habitan una casa no son los dueños de ella, sino que son prestadas por un pariente y generalmente no pagan ningún arriendo.

\section{(5) ASPECTOS SOCIO-CULTURALES}

\subsection{Familia, matrimonio y parentesco.}

Según la investigación el promedio de la familia, es de 8 a 9 personas, incluyendo las dos generaciones de padres e hijos. 
La vida matrimonial comienza temprano, es muy frecuente los casos en los cuales la mujer ha tenido su primer bebé a los 16 o 17 años de edad; por esta razón no es raro que una mujer de 35 años cuente con su segunda generación.

A pesar de existir el programa de planificación familiar que llega hasta la zona, no hay la acogida favorable de los habitantes de la misma.

Respecto a la convivencia marital, pese a no presentarse una marcada preferencia por los individuos de la misma comunidad, sin embargo se miran con agrado los matrimonios entre familias del lugar (véase: 5.1.1.).

La forma de convivir es la unión libre a la que denominan forma de vida "ajuntada". Sólo pocas parejas son casadas oficialmente, es decir con ceremonia civil y religiosa y algunos lo hicieron luego de vivir varios años en unión libre.

Existen razones tanto objetivas como subjetivas para este hecho: muchas veces faltan los documentos necesarios para casarse y otros afirman que "es muy largo y molestoso el trámite". Finalmente otros señalan que "el manabita si prefiere casarse, pero al esmeraldeño no le gusta mucho".

En general esta forma de unión tiene su tradición en la costa y el gobierno actual del Ecuador ha legalizado estas uniones, constituyéndolas legítimas sociedades conyugales. Naturalmente la Iglesia, no está de acuerdo con él y ha tratado de romper esta tradición pero con muy poco éxito.

Por otra parte se debe reconocer que casarse luego de varios años de unión libre, puede tener sus ventajas, ya que los largos años de relación menos obligatoria facilita una fluctuación entre los cónyuges, en donde la decisión de matrimonio es el fruto de la maduración afectiva.

La resistencia al matrimonio se debe a la costumbre, especialmente del hombre, de abandonar a su mujer y juntarse con otra. En todo caso no es fácil determinar desde el punto de vista sociológico, si esta costumbre es causa o efecto de la inestabilidad matrimonial. Existen casos en donde se pasa del segundo "ajuntamiento", pero también existen otros, en donde esta simple relación encierra fidelidad de toda una vida. 
Por falta de la obligación impuesta por la ley, la separación se realiza sin mayor dificultad. En esta situación los hijos normalmente se quedan con la madre, el padre en cambio puede contribuir al sustento de ellos en forma ocasional, dependiendo en todo caso de la relación afectiva que pueda haber todavía, de la situación económica y lógicamente de su nuevo compromiso con otra mujer.

Los hijos del primer compromiso de la mujer, en caso de haberlos, son aceptados por el nuevo marido; especialmente si son menores de edad son considerados como propios.

Puede observarse además que en el primer compromiso de la mujer, generalmente el hombre es mayor que ella; en cambio, en la segunda unión de la mujer, el hombre suele ser más joven. Este fenómeno se debe al sobrante masculino en la zona.

Esta situación hace difícil al hombre la búsqueda de una mujer joven en su comunidad y a veces la única alternativa que se le presenta es juntarse con una mujer mayor que él, abandonada y con hijos, quien por su parte, ante la perspectiva de una nueva protección masculina y una cierta seguridad respecto a la mantención de sus hijos, acepta sin mayores exigencias un nuevo compromiso de unión libre.

No obstante a lo mencionado anteriormente, lo común es la familia nuclear, compuesta por los padres e hijos y a veces extendida por una u otra persona más ya sea pariente/como en el caso de la abuela, el tío, o la prima; o simplemente un "arrimado" como el hijo adolescente de una familia amiga, un anciano amigo o un "hijo regalado".

Este último caso se da en los hogares sin hijos, generalmente cuando los esposos han llegado a una edad avanzada, aceptan gustosos al hijo que les ofrece una madre soltera o que proviene de hogares destruidos. Este convenio es muy beneficioso para las dos partes el niño regalado a un hogar sin hijos recibe toda la atención durante su crecimiento, pero al mismo tiempo, al llegar a ser joven, se convierte en el único sostén de sus ancianos "padres".

Normalmente existe una relación de parentesco entre los pobladores del recinto, pero también hay familias recién llegadas y por lo mismo sin lazos de parentesco con los demás. Del mismo modo, por la naturaleza del trabajo de jornaleros, en ciertas épocas del año llegan grupos de hombres solteros y hasta familias que solamente permanecen una temporada. 


\subsubsection{Preferencia marital, residencia y movilidad}

El estudio de los 51 informantes principales demuestra que tanto hombres como mujeres siguen viviendo en el mismo lugar de nacimiento y los que vienen de otros lugares están en proporción igual hombres y mujeres, a excepción de los pocos casos de personas llegadas de Guayaquil y Loja con predomino del sexo masculino.

Así mismo los investigados afirman que prefieren el matrimonio entre vecinos y conocidos del mismo lugar, manifestándose la natural reserva propia de una comunidad tradicional frente al forastero, especialmente ante la posibilidad de matrimonio.

Este hecho puede ser una reacción inconsciente frente a la gran inestabilidad del matrimonio (o de la libre unión), ya que afirman que el hombre forastero, sin una relación social tradicional con la comunidad, fácilmente puede marcharse del lugar abandonando a la mujer y sus hijos; inclusive luego de haber terminado con el patrimonio familiar o con la herencia de la mujer.

De acuerdo con la preferencia de formar una familia nuclear, normalmente el hombre al casarse construye su casa propia, en lo posible cerca de la casa de sus padres. La mujer, en cambio, abandona su familia para vivir en el lugar escogido por el marido.

Esta actitud adoptada por los nuevos esposos es la tradicional y más frecuente; sin embargo, como excepción se dan nuevas formas de enlace respondiendo a hechos y situaciones socio-económicas. En todo caso, el hombre goza de mayor libertad y por lo general es él, quien rompe la relación con su familia, para establecer un nuevo enlace en otro lugar.

Para tomar la decisión de "quedar o irse", se consideran factores como: la situación económica, la tenencia de tierras, bienes, empleo, etc. En este contexto la situación de los hijos tiene muy poca importancia. Esto especialmente en los matrimonios jóvenes.

Por otra parte no existe una tendencia a emigrar muy lejos, sino de principio se procura buscar un trabajo, adquirir un terreno, etc., en la misma región. 


\subsubsection{Sucesión y posesión de bienes}

En general la sucesión de bienes se realiza de acuerdo con la respectiva ley ecuatoriana. En los frecuentes casos de separación de los padres, los hijos siguen viviendo con la madre y a la muerte de ésta, heredan sus bienes (si es que los tiene). En cambio la herencia de los bienes del padre, depende de la relación existente entre éste y sus hijos. Por lo general sus pocos bienes únicamente heredan los hijos de la última unión.

Respecto a la posesión de las tierras, sólo hay pocas personas que cuentan con un título oficial. La mayoría ocupa su terreno por "derecho de posesión", a través de 2 o 3 generaciones.

\subsection{Organizaciones comunales.}

El principal organismo de la comunidad es la "Junta Pro Mejoras", integrada en su totalidad por personas de la comunidad y que se renuevan periódicamente. Este organismo es el encargado de velar por el progreso del recinto, centrando gran parte de sus esfuerzos al desarrollo del "Centro Poblado".

Sus actividades se orientan a la educación de los jóvenes, alfabetización de los adultos y el mejoramiento de las condiciones de salubridad, como sus preocupaciones fundamentales.

Otra de sus actividades en los últimos años, ha sido la realización de los trámites pertinentes para lograr que los terrenos ocupados por la sociedad ganadera Sánchez Merisalde, pasen a poder de la comunidad, alcanzándose plenamente este objetivo. Estas tierras que ahora son de la comunidad, necesitan de relleno por las frecuentes inundaciones.

Además la Junta Pro Mejoras, señala ciertas normas que deben acatar los moradores en la construcción de sus viviendas; como también se preocupa de la integración de las personas recién llegadas a la comunidad.

Como proyectos para el futuro, este organismo trata de lograr la instalación de una fábrica de enlatados de palmito o una industria de hielo, como también la instalación de una planta para secar y almacenar cacao; este último proyecto cuenta con el apoyo de OCAME (Organización Campesina de Muisne y Esmeraldas). 
A los asuntos comunales se dedica también el llamado "Grupo de Trabajo", en el que figuran como miembros casi las mismas personas que integran la Junta Pro Mejoras. Esta agrupación tiene el carácter de una pequeña cooperativa agrícola, los socios colaboran mutuamente en la preparación de la tierra, en la siembra, cosecha y comercialización de los productos y en otras cuestiones agrícolas.

Finalmente en Same funciona un Club Deportivo que participa activamente en los campeonatos de Fútbol e Indor, con otros recintos y parroquias de la Provincia.

\subsection{Vida Religiosa}

En su gran mayoría los moradores de Same son católicos y tratan de vivir la fe cristiana heredada de sus padres, naturalmente con todas las limitaciones propias dé sus costumbres y tradición.

Por lo general el párroco viene a la comunidad todos los domingos a celebrar la Misa y más oficios religiosos. Las fiestas litúrgicas se celebran aparentemente con menos fervor que en los tiempos pasados. Cuando una solemnidad religiosa cae entre semana, se traslada para el domingo siguiente, se celebra con sencillez y sin los priostes acostumbrados hasta hace algunos años.

En esta investigación no se ha podido detectar ningún vestigio de religiones africanas, pese a la visible influencia de la raza negra en el fenotipo de la zona.

También los evangélicos norteamericanos desarrollan una labor misionera en la zona, pero al parecer no tienen éxito; porque a más de unas cuatro familias prosélitas, en el resto de las familias no existe ninguna animosidad por abandonar el credo católico.

Los pastores protestantes tienen su reunión-una vez por semana (miércoles) para celebrar el culto. Han instalado un taller de carpintería en el que trabajan ocasionalmente y al momento de la investigación están construyendo una casa de retiros en la playa. 


\subsection{Fiestas y costumbres}

Hasta hace pocos años, según nos informan, la fiesta principal del recinto se celebraba el 16 de Julio, día de la Virgen del Carmen. Además de la Misa, se realizaba un desfile de antorchas, presentación de disfraces, cena común y baile popular; todo esto se organizaba cada año bajo la responsabilidad de la misma prioste, pero desde que esta señora se convirtió a la religión evangélica, abandonó esta costumbre y prácticamente desapareció esta fiesta.

Otras fiestas litúrgicas celebradas en la comunidad son: 1ro. de Junio, Fiesta del Corazón de Jesús; 29 de Junio, San Pedro y San Pablo; 13 de Junio, San Antonio; 20 de Noviembre la fiesta de Cristo Rey y el 30 de Agosto, Santa Rosa, celebrada especialmente en Atacames a donde acuden los moradores de Same.

Por lo demás las fiestas celebradas a nivel nacional y mundial, como la Navidad, el Año Nuevo, Carnaval y Semana Santa, se celebran al igual que en los demás pueblos. Sin embargo, vale aclarar que ninguna fiesta y en ningún momento, se celebra al estilo de la Sierra (con tal solemnidad y derroche de recursos).

En cuanto a las fiestas cívicas, se conmemoran los acontecimientos nacionales, más las efemérides de la Capital de la Provincia, el 5 de Agosto y el día de la parroquialización de Tonchigüe, el 26 de Noviembre. Estos acontecimientos se celebran, entre otros actos, con el desfile cívico, en el que participa la escuela de Same.

Un efecto negativo, cuestionado por los moradores del recinto, produce en la organización de cualquier fiesta, el hecho de que los actuales profesores no residen en el lugar de trabajo. Esto dificulta naturalmente la preparación de cualquier acto cultural o social. Los habitantes del lugar recuerdan con añoranza los tiempos en que los profesores residían en el lugar y contaban con el tiempo suficiente para la organización y preparación de los actos de las festividades.

También se celebran las fiestas familiares, como bautismos, onomásticos, etc. En los pocos casos en los que se realizan matrimonios; son muy celebrados, luego del acto civil o litúrgico, vienen los bailes, comida y bebida abundantes. 
La muerte de un individuo, en la mayoría de los casos, también es ocasión de borracheras, ya que en el velorio se sirve abundante aguardiente $\mathrm{y}$, de la misma manera, al día siguiente en el entierro. Después vienen los nueve días (novena) de reuniones y oraciones por el difunto que se realizan por las noches en la casa del duelo.

La velación de un niño difunto, se denomina también "anillo" y se realiza en forma parecida a la acostumbrada por la población negra de la región de San Lorenzo (véase Whitten, Class, Kinship, and Power in an Ecuadorian Town, 1965, pp. 143), pero según las informaciones, en Same se realiza en forma más sencilla y menos pintoresca que en San Lorenzo.

\subsection{Música y Danza}

Los ritmos más populares en Same, al igual que en toda la provincia de Esmeraldas, son los obtenidos con instrumentos de percusión como : bombos, guasas, canutos y maracas, se nota en ellos el fuerte influjo de la raza negra.

Entre las melodías típicas de la provincia están las danzas con marimbas: chigualo, currulao, arullo, etc. Además de éstas, se interpretan las canciones del folklore nacional: pasillos, sanjuanitos, etc., y las salsas, guarachas y cumbias, típicas de los ambientes tropicales.

Naturalmente en las canciones populares no puede faltar el acompañamiento de la guitarra.

Sin embargo, en Same no existe ningún conjunto o agrupación musical, razón por la cual los moradores del lugar contratan conjuntos o bandas en la ciudad de Esmeraldas para sus fiestas locales; pero por el elevado costo de estas agrupaciones musicales, la mayoría de las veces se utiliza únicamente los equipos de sonido existentes ya en el lugar.

\subsection{Tradición oral: cuentos y leyendas.}

Existe una tradición oral que no es exclusivamente de la comunidad de Same, sino que es conocida en casi toda la provincia y ha sido transmitida de padres a hijos a través de varias generaciones.

También se conocen leyendas y supersticiones en las que todavía creen de buena fe, la mayor parte de los moradores del recinto. Según esto, seres fantásticos y fuerzas sobrenaturales actúan con su poder invencible en 
la vida cotidiana de los seres humanos. (Versión del Sr. Carlos Olivo Vera, véase también García-B. 1981 pp. 543).

Algunas de las principales leyendas son:

\section{La Tunda:}

Es un ser extraño que se presenta a determinadas personas con el objeto de conquistarle y llevarle consigo; no tiene predilección por los jóvenes; le basta que sean hombres y que se dejen conquistar, sea joven o viejo da igual.

Cuando se lleva a la víctima, la Tunda va delante soltándose muchos pedos que le emboban al hombre. En el camino coge camarones los pasa por debajo de su falda, sacándoles bien asados para dárselos de comer al "entundado".

Algunas veces deja en el camino huellas de unos pies "milinillos"; tiene dedos alrededor de todo el pie, por eso le dicen "pata de molinillo".

\section{El Duende:}

Se presenta en forma de hombrecillo con un sombrero muy grande, se comporta muy enamorado de las mujeres esbeltas y bonitas, lanza unos silbidos que es su forma típica de comunicarse con la mujer que está enamorado. Además de esto la mujer se da cuenta de su presencia, aunque todavía no lo vea porque si en ese instante ella desea algo, por ejemplo desea algún manjar o una prenda de vestir, al instante "caen" en la habitación donde se encuentra ella.

Cuando la chica se da cuenta que el duende está enamorado de ella, para decepcionarlo tiene que simular ser muy desordenada y "cochina", así por ejemplo llevar algo de comer al baño, mezclar los alimentos con desperdicios y comérselos, etc. Ante estas actitudes, el duende sufre tal decepción que jamás la vuelve a silbar ni hacer que se cumplan los deseos de la chica, así ésta se ve libre de él definitivamente.

Se afirma también que el duende era el .mejor músico del coro celestial y que fue arrojado del paraíso igual con Luzbel; por esta razón, hay otra forma de ahuyentarlo: cuando se conoce que él frecuenta un lugar se le 
deja allí una guitarra, él llega y la toca y se a- cuerda que un tiempo fue muy feliz en el paraíso siendo ángel; al instante rompe la guitarra y no vuelve más a ese lugar.

\section{La Gualgura:}

Debe tener la forma de un pollo, ya que a veces se le oye piar por las noches en el monte; mucha gente ha sido engañada al ir a buscarla, creyendo encontrar gallinas perdidas.

Este ser es considerado como visión maligna y trae la peste a las aves, especialmente a los pollos pequeños. Para ahuyentarla, se da golpes con la piedra de moler, al tiempo que se grita: "volverás por sal".

\section{El Riviel:}

Tiene la figura de un potrillo, con una lucecilla en la frente, nada en las desembocaduras de los ríos o en las ensenadas, acude de inmediato cuando escucha llorar un niño con el propósito de llevarlo consigo. Esto se debe, según la leyenda, a que el Riviel se originó de una madre que no queriendo tener a su hijo, lo arrojó al agua, por lo que Dios le castigó ordenando traer al niño o por lo menos todos sus huesos. De ahí que cuando escucha llorar un niño quiere arrebatarlo y llevarlo consigo.

\subsection{Indumentaria}

En la indumentaria usual, no acostumbran ningún elemento tradicional, ni folklórico; se demuestra únicamente la preferencia, tanto en hombres como en mujeres, por los colores intensos y brillantes.

El hombre lleva un pantalón de algodón o de fibras sintéticas, a veces también un calentador deportivo; una camiseta con letras y dibujos impresos o una camisa multicolor; como calzado, unas sandalias o zapatos deportivos; para ir al desmonte utilizan botas de caucho. El traje se completa con un sombrero de paja o tela de alas anchas o una gorra de béisbol.

En el caso de la mujer, la indumentaria se compone de un vestido o una blusa y falda; todo de colores intensos y llamativos; a veces utilizan las sandalias u otras veces van descalzas. 
Como traje festivo el hombre se reserva al menos un pantalón y una camisa; la mujer también tiene su vestido extra a colores sea floreado o con rayas. Las jóvenes usan tacones altos para el paseo del domingo o para los bailes.

Las mujeres se adornan con collares, aretes, brazaletes de oro, plata y más frecuentemente de bambalina. Los cabellos lo adornan con prendedores multicolores.

Toda su indumentaria la adquieren en los mercados de las ciudades. En Same no existen talleres de sastrería, ni modistería; son pocas las familias que disponen de una máquina de coser, dándole únicamente una función doméstica.

\subsection{Alimentación}

Los moradores de Same, al igual que lo hace toda personaje alimentan tres veces al día.

El desayuno, en la mayoría de los casos, consiste en café con banano; en algunas familias se agrega en el desayuno una porción de pescado, o unos huevos fritos con arroz; pero también en otros hogares muy pobres, todo se limita a una porción de banano con agua dulce.

El almuerzo generalmente se compone de sopas de verduras y otros componentes; el arroz que no puede faltar ya sea con pescado, o alguna clase de carnes.

La merienda por lo general es parecida en sus platos al almuerzo; claro que en menor cantidad. En todo caso el arroz y las verduras son componentes que jamás pueden faltar en la ración alimenticia de esta comunidad, juntamente con las abundantes frutas de la zona.

En el caserío no existe una panadería, el pan viene de las poblaciones vecinas y resulta bastante caro, razón por la cual se lo consume muy poco.

Como bebidas: el agua natural, jugos de frutas, el agua de coco y agua de panela, el café es menos frecuente en la zona. Por el precio relativamente elevado, muy pocos consumen las gaseosas que se expenden en las pocas tiendas del lugar. 
Como bebidas alcohólicas, se expenden aguardientes de diferente calidad, el más común el "Cristal", también el "Frontera", el "Cayapa" y el "Auca". Además se expende la cerveza que por su elevado costo no puede ser consumida por muchas personas.

Por lo general son los fines de semana cuando se consumen las bebidas alcohólicas, ya que durante la semana el único establecimiento de este tipo que hay en la población permanece cerrado, porque su propietario se dedica a las labores agrícolas.

En ocasiones festivas la comida también es especial; se compone de una gran variedad de platos, entre ellos: caldo de camarones, aguado de gallina, sancocho de pescado, langostinos, carnes de cerdo, res, pollo, etc., además del arroz y banano. Y como es natural no pueden faltar las bebidas alcohólicas.

La alimentación de los infantes se la realiza con la leche materna o con la leche de vaca; posteriormente se los administra coladas a base de maíz o banano, mezcladas con jugos de frutas. El uso de leches procesadas, así como de cereales fabricados, no tiene importancia en la comunidad de Same; seguramente por razones económicas.

A los niños de corta edad, ya se los acostumbra a la comida de los adultos. Uno u otro alimento tienen su temporada; por ejemplo el maíz, langostinos y camarones se acostumbra más en los meses de Marzo y Abril; los cangrejos son más abundantes en los meses de Noviembre y Diciembre y se obtienen en el estero vecino.

\subsection{Educación}

La escuela originariamente situada en la playa, como se ha dicho, fue trasladada junto con las demás viviendas al nuevo centro de Same, en donde funciona actualmente con jornada matutina de $7.30 \mathrm{~h}$. a $12.30 \mathrm{~h}$. de lunes a viernes. Allí laboran dos maestras y un practicante que vienen diariamente de la ciudad de Esmeraldas.

Los padres de familia reclaman mayor responsabilidad de los maestros, que con frecuencia faltan o llegan atrasados, porque no residen en el lugar y tienen que realizar un largo viaje diariamente.

Por su parte los profesores, no aceptan este reclamo y argumentan que la falta de éxito en la labor educativa en el recinto se debe a la falta de 
colaboración de los padres de familia, que no mandan regularmente a sus hijos a la escuela, argumentando que no tienen para los uniformes o para los útiles escolares que se solicitan. En definitiva con esto demuestran los padres de familia la poca importancia que dan a la educación de los hijos.

También afirman los profesores que muchos alumnos vienen solo una temporada; en otras ocasiones la movilización continua de padres hace que los hijos tengan que abandonar la escuela, impidiéndose una formación permanente y eficaz.

Para la instrucción secundaria, los jóvenes tienen que trasladarse diariamente a la parroquia de Tonchigüe, en donde funciona un colegio. Ya sea por la situación económica o por la falta de conciencia de la necesidad de la educación, todavía son muy escasos los padres de familia qué envían a sus hijos al colegio.

En todo caso los datos estadísticos demuestran que de los 81 hombres investigados, sólo 5 de ellos llegaron a las aulas del colegio, de los cuales uno logró culminar el sexto curso, otro se retiró luego del cuarto curso, dos hicieron hasta el segundo y uno se retiró apenas concluido el primer año de bachillerato.

Los restantes 76 hombres investigados se reparten en el siguiente cuadro:

\begin{tabular}{|c|c|c|c|c|c|c|c|c|}
\hline $\begin{array}{c}\text { Edad } \\
\text { años }\end{array}$ & $\begin{array}{c}\text { Sin } \\
\text { Educ. }\end{array}$ & 1.gr. & 2.gr. & 3.gr. & 4.gr. & 5.gr. & 6.gr. & $\begin{array}{c}\text { Total } \\
\text { por edad }\end{array}$ \\
\hline $10-19$ & 2 & 0 & 4 & 0 & 0 & 0 & 5 & 11 \\
\hline $20-29$ & 1 & 1 & 0 & 2 & 0 & 0 & 10 & 15 \\
\hline $30-39$ & 3 & 1 & 3 & 1 & 5 & 1 & 3 & 17 \\
\hline $40-49$ & 6 & 0 & 1 & 6 & 2 & 2 & 1 & 18 \\
\hline $50-59$ & 1 & 0 & 0 & 0 & 1 & 1 & 2 & 5 \\
\hline $60 \mathrm{y}+$ & 4 & 2 & 2 & 0 & 2 & 0 & 0 & 10 \\
\hline $\begin{array}{c}\text { Total } \\
\text { por gr. }\end{array}$ & 17 & 4 & 10 & 9 & 11 & 4 & 21 & 76 \\
\hline
\end{tabular}

Como se puede apreciar en este cuadro, es evidente que las nuevas generaciones tienen mayores posibilidades de educación, sin embargo del grupo de los 10 a los 19 años sólo el $50 \%$ han terminado la primaria. Por otra parte el grupo de los 50 a los 59 años, apenas consta en este diagrama.

Con respecto al nivel educativo de las mujeres, se presentan los siguientes resultados: de las 76 personas investigadas, 9 llegaron a recibir instrucción secundaria, terminando este nivel solamente una de ellas. 
De las restantes, una aprobó el quinto curso, dos abandonaron el colegio luego del cuarto año, una aprobó hasta el tercero, dos hicieron solamente hasta el segundo y tres apenas lograron aprobar el primer año de bachillerato.

En la instrucción secundaria se puede apreciar una considerable ventaja de las mujeres con respecto a los hombres, en cuanto al número; esto se debe a que posiblemente los varones a muy temprana edad, ya tienen que contribuir con su trabajo para la subsistencia de la familia. En cambio parece que las mujeres tienen más posibilidad de estudiar mientras permanecen con sus padres, pero se les cierra esta oportunidad con el matrimonio, al que llegan relativamente pronto.

En lo que respecta a la educación primaria, no existe una diferencia significativa entre los dos sexos, como se demuestra en el siguiente cuadro.

\begin{tabular}{|c|c|c|c|c|c|c|c|c|}
\hline $\begin{array}{l}\text { Edad } \\
\text { Años }\end{array}$ & $\begin{array}{l}\text { Sin } \\
\text { Educ. }\end{array}$ & 1. gr. & 2.gr. & 3. gr. & 4. gr. & 5. gr. & 6. gr. & $\begin{array}{c}\text { Total } \\
\text { Por edad }\end{array}$ \\
\hline $10-19$ & 0 & 0 & 0 & 1 & 0 & 0 & 0 & 1 \\
\hline $20-29$ & 2 & 2 & 2 & 2 & 4 & 5 & 8 & 25 \\
\hline 30-39 & 1 & 3 & 2 & 1 & 1 & 1 & 8 & 17 \\
\hline $40-49$ & 3 & 0 & 3 & 4 & 1 & 1 & 1 & 13 \\
\hline $50-59$ & 2 & 0 & 0 & 1 & 0 & 0 & 1 & 4 \\
\hline $\begin{array}{l}60 \mathrm{y}+ \\
\text { Total }\end{array}$ & 5 & 0 & 1 & 1 & 0 & 0 & 0 & 7 \\
\hline Por gr. & 13 & 5 & 8 & 10 & 6 & 7 & 18 & 67 \\
\hline
\end{tabular}

En el grupo de personas que actualmente están en la edad escolar obligatoria o que siguen sus estudios en los distintos niveles, se encuentran 100 personas (50 hombres y 50 mujeres).

\begin{tabular}{|c|c|c|c|c|c|c|c|c|c|c|c|c|c|c|c|c|c|c|}
\hline HOMBRES & & PRIM & ARI & & & & & & $\mathrm{CU}$ & DA & RIA & & & & SUPE & IOI & & TOT. \\
\hline Ed s/ed & 1 & 23 & 4 & 5 & 6 & $\mathrm{~T}$ & 1 & 2 & 3 & 4 & 5 & 6 & $\mathrm{~T}$ & 1 & 23 & 4 & $\mathrm{~T}$ & $\mathrm{~T}$ \\
\hline $6-9 \quad 1$ & 8 & 52 & & & & 16 & & & & & & & & & & & & 16 \\
\hline $10-14$ & 4 & 14 & 4 & 2 & 1 & 17 & 1 & & & & & & 1 & & & & & 18 \\
\hline $15-19$ & $1 *$ & 2 & & 1 & 2 & 5 & 4 & 2 & 1 & 1 & 1 & & 9 & & & & & 14 \\
\hline $\begin{array}{l}20-29 \\
30+\end{array}$ & & & & & & & & & & & & 1 & 1 & & & 1 & 1 & 2 \\
\hline TOTAL 1 & 13 & 68 & 4 & 3 & 3 & 38 & 5 & 2 & 1 & 1 & 1 & 1 & 11 & & & 1 & 1 & $\mathbf{5 0}$ \\
\hline
\end{tabular}




\begin{tabular}{|c|c|c|c|c|c|c|c|c|c|c|c|c|c|c|c|c|c|c|c|}
\hline MUJERES & & & MA & RIA & & & & & SECL & UND & ARIA & & & & & ER & IOR & & \\
\hline dd $\quad s / e d$ & 1 & 2 & 3 & 4 & 5 & 6 & $\mathrm{~T}$ & 1 & 23 & 34 & 5 & 6 & $\mathrm{~T}$ & 1 & 2 & 8 & & $\mathrm{~T}$ & \\
\hline-9 & 9 & 4 & 3 & & & & 22 & & & & & & & & & & & & 2 \\
\hline $0-14$ & 1 & 2 & 4 & 4 & 2 & 3 & 16 & 2 & & & & & 2 & & & & & & \\
\hline $5-19$ & & & & & 1 & & 1 & 2 & 1 & 2 & & 1 & 6 & & & & & & \\
\hline-29 & & & & & & 1 & 1 & & & & & & & 1 & & & & & \\
\hline $0+$ & & & & & & & & & & & & & & & 1 & & & & \\
\hline OTAL 6 & & 10 & & 7 & 4 & 3 & 4 & 40 & $4 \quad 1$ & 1 & 2 & & 1 & 8 & 1 & 1 & & 2 & \\
\hline
\end{tabular}

Estas estadísticas también nos muestran la clara tendencia declinante respecto al avance en los niveles secundario y superior, sin diferencias significativas entre los dos sexos; la instrucción superior apenas está presente en este cuadro.

Por otra parte, se puede observar a veces una gran discrepancia entre la edad y el nivel de estudios (grado o curso) de la persona; esto se explica fácilmente por diversos factores entre ellos el principal: la difícil situación socio-económica propia de esa comunidad. Además, los matrimonios tempranos, las frecuentes movilizaciones de la familia, etc. son factores que dilatan, interrumpen o impiden la realización de un proceso educativo en forma oportuna y adecuada.

Del total de investigados 37 personas (18 hombres y 19 mujeres) son analfabetas, lo cual da un porcentaje del $14 \%$. Si se considera que muchas personas que apenas llegaron al primero o segundo grado de la primaria, están en peligro de volver a ser analfabetas por regresión, la situación se agrava todavía más.

En un estudio sobre educación realizado por la OEA (El déficit Educativo en América Latina, 1979), se señala el porcentaje de 15,9 \% de analfabetismo en el Ecuador y considerando únicamente la provincia de Esmeraldas, el porcentaje se eleva al $22,9 \%$, con respecto a la población entre los 6 y 24 años. Claro está que los datos para este estudio fueron tomados del censo de 1974 y naturalmente desde esa fecha hasta el momento de la actual investigación, las cosas han cambiado.

\section{(6) SALUD}

A pesar de que los moradores del lugar, consideran que gozan de un buen estado de salud, sin embargo, se hacen presentes las enfermedades que provienen de la falta de prevención higiénica. Se han presentado casos de tifoidea y otras infecciones intestinales causadas por bacterias, virus y parásitos. 
También se han dado varios casos de paludismo, aunque con consecuencias menos desastrosas que en décadas pasadas.

En los niños las enfermedades más frecuentes son: bronquitis, tosferina, sarampión, viruela, tuberculosis, tétano y enfermedades parasitarias. Estas enfermedades son la causa de una elevada tasa de mortalidad infantil, sobre todo en los menores de 6 años.

Por otra parte se debe mencionar ciertas enfermedades que para la gente del lugar se quedan en un ambiente semimágico; por ejemplo el "mal ojo" llamado también "el mal humor", el "mal de los 7 días", etc. Estos males son tratados por el curandero y a veces aceptados con un fatalismo total como algo irreversible, sin averiguar las causas reales.

A continuación el cuadro estadístico de mortalidad infantil:

\begin{tabular}{|c|c|c|c|c|c|}
\hline $\begin{array}{l}\text { Edad las } \\
\text { Madres } \\
\text { Investig. }\end{array}$ & $\begin{array}{l}\text { Número } \\
\text { de mujeres } \\
\text { investig. }\end{array}$ & $\begin{array}{l}\text { Número } \\
\text { de } \\
\text { embarazos }\end{array}$ & $\begin{array}{l}\text { Promedio } \\
\quad \text { de } \\
\text { hijos/mujer }\end{array}$ & $\begin{array}{c}\text { Número } \\
\text { de } \\
\text { muertes }\end{array}$ & $\begin{array}{l}\% \text { de } \\
\text { mortal. }\end{array}$ \\
\hline 24 años y - & 3 & 10 & 3.3 & 1 & $10 \%$ \\
\hline 25-29 años & 6 & 30 & 5 & 9 & $30 \%$ \\
\hline 30-34 años & 5 & 41 & 8 & 3 & $7.3 \%$ \\
\hline 35-39 años & 8 & 53 & 6.5 & 7 & $13.2 \%$ \\
\hline 40-44 años & 9 & 73 & 8 & 11 & $15 \%$ \\
\hline 45-49 años & 0 & - & - & - & - \\
\hline 50-54 años & 1 & 2 & 2 & 0 & - \\
\hline $55-59$ años & 4 & 32 & 8 & 5 & $15.6 \%$ \\
\hline 60-64 años & 1 & - & - & - & - \\
\hline 65 años y + & 3 & 27 & 9 & 14 & $51.9 \%$ \\
\hline TOTAL & 40 & 268 & 6.7 & 50 & $18.6 \%$ \\
\hline
\end{tabular}

En el total de muertos constan también los abortos y los niños nacidos muertos. Sin embargo la mayor parte corresponde a los niños muertos antes de cumplir el primer año de vida (del total de 50 corresponden 44).

De lo anterior se deduce que el primer año, resulta el tiempo más difícil en la crianza de los niños. El porcentaje corresponde al $88 \%$ del total de mortalidad infantil, lo que representa el 18,6\% del total de embarazos registrados. 
Son 141 niños entre 1.000 nacidos vivos, que han fallecido en el primer año de vida. Este dato difiere de las estadísticas oficiales a nivel nacional que señalan el porcentaje de 71.5, sin indicar la edad de los niños (véase almanaque mundial 1982).

No hay duda de que los datos recogidos en esta comunidad, reflejan más la realidad que los datos oficiales; porque contienen todos los fallecimientos de acuerdo con la afirmación de los padres; mientras que en las estadísticas oficiales sólo constan los fallecimientos puestos en conocimiento del médico, hospital y Registro Civil, cosa que normalmente ocurre muy poco, debido a los factores socio-económicos del lugar.

Sin embargo, parece que la situación actual al respecto ha mejorado notablemente, en comparación con la generación de las madres que hoy alcanzan los 65 años y más.

\subsection{Alcoholismo}

Si bien el problema del alcoholismo no es tan grave como en ciertas regiones de la Sierra, tampoco podemos afirmar que se halla ausente en esta comunidad.

No existen alcohólicos "adictos"; según informes de los moradores, en cada familia sobresale algún miembro "inclinado" al alcohol pero no dominado por el vicio. Generalmente los hombres beben licores en los fines de semana y naturalmente en las fiestas locales y familiares en donde también participan las mujeres, aunque en menor escala que los varones.

En ocasiones bajo los efectos del alcohol, afloran los sentimientos de celos y rencores, ocasionando disgustos y peleas callejeras.

El siguiente dato proporcionado por los dueños de tiendas y cantinas nos demuestra que se consume una considerable cantidad de bebidas alcohólicas: en un mes las tiendas venden alrededor de 30.000 sucres; de esta suma aproximadamente la mitad corresponde a bebidas alcohólicas y si consideramos el total de los ingresos por familia, el $10 \%$ de éstos se destinan al consumo de estas bebidas. 


\subsection{Consumo de drogas}

La cercanía a los centros turísticos de Atacames y Súa, en donde comercian los narcotraficantes locales y nacionales con la clientela internacional, afecta a la comunidad de Same de una u otra manera.

Sin embargo según afirmación de muchos de los moradores del lugar, en los últimos años ha disminuido considerablemente este problema, debido a las denuncias realizadas por los mismos vecinos del lugar a la policía.

\subsection{Defectos mentales}

De las 334 personas investigadas, existen tres jóvenes definidos por sus familiares y vecinos como "retardados", sin que se haya dado un diagnóstico médico al respecto.

Estas deficiencias se presentaron desde el nacimiento. Actualmente las dos mujeres tienen alrededor de 23 años y el joven 15 años. También existe, según información de los vecinos, una mujer de edad avanzada que actualmente no vive en Same y que demuestra tener problemas en su salud mental.

\subsection{Asistencia médica y problemas de salud}

Como ya se ha mencionado anteriormente la asistencia médica es considerada como insuficiente por los moradores de este recinto. En Tonchigüe existe un Subcentro de Salud, pero que no presta un servicio eficaz, según juicios emitidos por muchas personas: "No tenemos mucha confianza en el médico rural", "nunca está en el consultorio", "sólo atiende desde el carro".

Por otro lado la gente no está en la capacidad económica de costearse el tratamiento en los consultorios o clínicas de Atacames o Esmeraldas. Añádase a esto el alto precio de los medicamentos.

En consecuencia la gente sólo acude al médico en casos muy graves, generalmente cuando ya no se puede hacer nada por salvarlo. En los demás casos, prefieren los remedios caseros tradicionales, o llevarlo donde el 
curandero/a que trata con eficacia los llamados "males comunes" como el "mal ojo", el "mal de los 7 días", "fracturas", "espanto", etc.

Naturalmente el trabajo del curandero no es gratis y sus honorarios no se diferencian mucho de los exigidos por el médico, pero la gente tiene confianza en él y da la casualidad que está disponible a toda hora.

La preferencia por el curandero/a, comadrona, etc., es manifestación de la actitud tradicional frente al asunto de medicina y salud.

Así, es muy frecuente que los alumbramientos sean asistidos por parteras o comadronas autodidactas o simplemente por miembros de la misma familia y el parto se realiza en la propia casa. Sin embargo, últimamente en los hogares jóvenes hay la preferencia por llevar a la parturienta al médico o al hospital, este cambio de actitud se debe en gran parte a la facilidad de movilización desde Same a las ciudades vecinas.

Hay que considerar que no obstante a que el parto tradicional predomina todavía en esta comunidad, no se han presentado casos de mortalidad maternal, por ejemplo como resultado de fiebre puerperal.

En lo relativo a enfermedades y epidemias corrientes, no existe una conciencia general hacia una adecuada profilaxia. Así, a pesar de los programas oficiales de salud, no son muy frecuentes los casos de vacunas contra enfermedades infantiles.

Contra el paludismo los representantes del Ministerio de Salud suministran medicamentos a los enfermos y como profilaxia fumigan las casas, chozas, esteros, etc. una vez al año.

Como es fácil deducir, el tratamiento una vez al año no es suficiente para eliminar las varias generaciones de mosquitos que se reproducen en este lapso; por otra parte los estanques permanecen como fuentes de infección y semilleros de insectos.

Además, el uso del DDT acarrea efectos nocivos a la salud del hombre, razón por la cual ha sido prohibido su empleo en Europa y Estados Unidos, sin embargo se sigue utilizando en los países del Tercer Mundo. Últimos estudios realizados por la $\mathrm{ONU}$ en comunidades indígenas del 
Oriente peruano, demuestran una vez más las graves consecuencias del uso del DDT.

Al fumigar, el polvo de acción penetrante se extiende sobre todo lo que encuentra a su paso: muebles, vestidos, vajillas y alimentos cuando no están lo suficientemente protegidos. Además es inevitable la penetración de este insecticida en el organismo humano a través del sistema respiratorio.

\subsubsection{Control de la natalidad}

Ya se mencionó anteriormente la presencia de una representante voluntaria del programa de planificación familiar en el recinto, pero con escaso éxito en su labor, ya que son muy pocos los casos de mujeres que consultan con ella y que utilicen los medios de control ofrecidos.

Además la aplicación se realiza en forma discriminada, ya que solamente la mujer se somete al control. Por otra parte existe la resección total del útero, pero no por razón social de control de natalidad sino por razones médicas.

\section{(7) ECONOMIA}

\subsection{Agricultura y tenencia de la tierra}

La actividad agrícola es la ocupación principal de los moradores de Same. Sin embargo la posesión de la tierra todavía no está legalizada en la mayoría de los casos.

De las 49 familias en cuestión, sólo 5 tienen títulos de propiedad; otras tres familias están pagando las cuotas al IERAC que está ya realizando los trámites de medición y entrega de terrenos. Los demás casos sólo se basan en su "derecho de posesión" y reclaman la legalización por parte de la institución correspondiente.

La extensión de los terrenos en posesión, varía desde un solar, hasta las fincas de 100 hectáreas; de lo cual sólo una parte es de terreno plano, el resto constituyen quebradas y montañas de poca elevación pobladas por exuberante vegetación tropical. 
En las fincas extensas, aproximadamente apenas un $10 \%$ están cultivadas, mientras que las propiedades hasta de 5 hectáreas están cultivadas en su totalidad, como se demuestra en el siguiente cuadro:

$\begin{array}{llc}\begin{array}{c}\text { Extensión } \\ \text { Terrenos }\end{array} & \begin{array}{c}\text { Nro. Entidades económicas } \\ \text { (familia) }\end{array} & \begin{array}{l}\text { Porcentaje } \\ \text { cultivado }\end{array} \\ \text { Sin tierra } & 14 & -------- \\ 0-1 \text { hectáreas } & 7 & 100 \% \\ 1-5 \text { hectáreas } & 10 & 90 \% \\ 5-10 \text { hectáreas } & 3 & 50 \% \\ 10-20 \text { hectáreas } & 3 & 18 \% \\ 20-30 \text { hectáreas } & 2 & 16 \% \\ 30-40 \text { hectáreas } & 2 & 10 \% \\ 40-50 \text { hectáreas } & 4 & 12 \% \\ -\quad 100 \text { hectáreas } & 4 & 5 \%\end{array}$

Los terrenos no cultivados en parte constituyen los llamados potreros, aunque no son utilizados en la ganadería.

Los productos que se dan en la región como el guineo, café, cacao, plátano, cocos, etc. se cultivan mezclados en una misma extensión de terreno, sin técnica alguna ni la modalidad del monocultivo como en otras regiones de la costa.

Para la agricultura se utilizan únicamente métodos y herramientas rudimentarias, principalmente el machete y el hacha. Además es poco frecuente el uso de fertilizantes y otros productos químicos adecuados, para mejorar la producción, esto debido a la falta de recursos económicos del pequeño agricultor.

De este modo la economía agrícola se basa en una cosecha bien distinta en calidad y cantidad de productos, dependiendo en gran parte de las condiciones climáticas y otros fenómenos naturales. Así por ejemplo, con respecto al café en el año 1983/84 casi no hubo producción alguna, debido a las lluvias excesivas en el tiempo de la floración.

Solamente los cultivos de banano y cocos, garantizan un cierto ingreso relativamente fijo y a veces compensa las pérdidas en las cosechas de café y cacao. 
En años anteriores, recuerda un agricultor que recogió unos 40 quintales de café, que los vendió a 900 cada uno, unos 30 quintales de cacao vendidos a 1.500 sucres cada uno y unos 75 quintales de maíz a razón de 270 sucres el quintal. Esta es considerada como una buena o excelente cosecha, como resultado del tiempo favorable y permanente cuidado.

En la actualidad el maíz se cultiva en cantidades relativamente pequeñas, debido al alto costo en la preparación de los terrenos y limpiezas posteriores. Así se estiman que estos gastos suman alrededor de 7.000 sucres por hectárea. Por el contrario, el desmonte para los cultivos de café o cacao, cuesta aproximadamente la mitad. Por esta razón con pocas excepciones, el maíz se cultiva únicamente para el consumo familiar.

El cultivo de verduras y frutas que se realiza alrededor de la casa, generalmente es trabajo de la esposa y los niños.

Como resultado de ese sistema económico limitado con respecto a la inversión de fondos y empleo de mano de obra, las ganancias anuales también son limitadas (cuando las hay). Algunos agricultores hablan de un superávit anual de 10.000 a 40.000 sucres por la venta de sus cosechas.

\subsection{Ganadería y cría de animales domésticos}

Existen alrededor de unos cuatro terratenientes que no viven en Same, pero que mantienen allí fincas extensas con ganado bovino.

De los moradores del lugar, únicamente cinco poseen ganado propio en número relativamente bajo: $4,15,22,37$ y 40 bovinos respectivamente.

Uno de estos propietarios trabaja al mismo tiempo como mayordomo en una de las fincas ganaderas con sueldo fijo (sueldo de un mayordomo de 3.000 a 5.000 sucres mensuales).

Una señora cuida en su terreno unas 18 reses, cuyos propietarios viven en Nueva York y pagan 30 sucres mensuales por cada cabeza de ganado. 
Las razas más comunes son el ganado Zebú y el Criollo, animales robustos propiamente de carne; en cambio no existe el tipo de ganado de leche, razón por la cual la producción de este alimento es escasa y no tiene ninguna importancia en la economía del lugar.

Las vacunas, alimentos balanceados y otros productos sólo se utilizan en las haciendas ganaderas; el pequeño propietario de ganado considera demasiado elevado el costo de esos productos que se suma a otros gastos, como el pago de las cuotas al banco por los préstamos para la adquisición de terrenos o más ganado.

Estos gastos se compensan naturalmente con la venta del mismo ganado, pero no siempre queda un superávit considerable; ya que muchas veces se presentan enfermedades, como por ejemplo la llamada "piponera", mal de ganado que causa la muerte de varias reses de un día para otro.

Según afirman, en condiciones normales el superávit anual en la crianza de unas 20 a 40 reses, alcanza hasta los 30.000 sucres.

En el tiempo de la investigación, afirman que el valor de una vaca "en pie" fluctúa entre los 15.000 y 25.000 sucres, es decir a un promedio de 5.000 sucres el quintal.

En conclusión, hay una enorme diferencia en inversiones, procedimientos y resultados, entre los grandes ganaderos y el pequeño propietario. Mientras los primeros obtienen fuertes ganancias, los segundos a veces ni siquiera compensan los gastos y esfuerzos empleados.

Entre otros animales domésticos se cuentan algunos ejemplares de ganado caballar (5 caballos, 2 mulares) y porcino (25) cerdos. Por el clima tropical es imposible la crianza de ovejas. Además en cada casa se observa una cantidad de gallinas, gansos y patos, que proporcionan huevos y carne para el consumo familiar.

\section{3. $\quad$ Pesca}

La temporada que normalmente se extiende desde Enero a Mayo es buena para la pesca de camarones ("pomada") y langostinos, que para algunas familias representa un ingreso económico considerable (7 familias). 
A la pesca de otras especies marítimas conocidas en la zona como la corvina, corona, pardo, robaldo, jural, pez sierra, tiburón, raya, etc., no se dedican los moradores de Same por falta de un equipo técnico adecuado, que resulta muy costoso.

Los moradores de Tonchigüe, que poseen lanchas lo suficientemente grandes y mejores equipos, si se dedican a la pesca en mayor escala, aunque la pesca de altura no se puede realizar en ninguna parte de esta región por la falta de un puerto hondo y con muelle.

Los pescadores de Same sólo disponen, de canoas (bongos) a remo. En el tiempo de la investigación sólo hubo un poco de pesca de camarones, aunque fuera de tiempo y por lo mismo con escasos resultados. Los pescadores sólo navegaban hasta unos 200 o 300 metros en el mar.

Además de las mencionadas 7 familias, unas 10 familias o más también se dedican a la pesca aunque ocasional y menos organizada. Por lo general estas personas no disponen de un equipo propio; a veces alquilan una canoa o trabajan en sociedad con pescadores que disponen de todo el equipo necesario.

Según información proporcionada por los mismos moradores del lugar, esta ocupación, que representa un considerable ingreso económico temporal, es nueva entre los habitantes de Same y ninguno se dedica exclusivamente a esta actividad, sino que son principalmente agricultores, carpinteros o empleados en las ganaderías vecinas.

Inicialmente la pesca por parte de los moradores de Same, tuvo el fin exclusivo de proveer de estos alimentos a la familia; pero la actividad se volvió rentable con el establecimiento de dos hoteles en la playa, así como también con la apertura de la nueva carretera que permite la comercialización de toda clase de productos.

En todo caso, en la actualidad, son altas las inversiones que se tienen que realizar para iniciarse en la actividad de la pesca de crustáceos. Se requiere de una canoa (bongo de cerca de tres metros de largo), que cuesta aproximadamente unos 4.000 sucres y por lo menos 4 o 6 redes aptas para camarones (lo normal es de 6 a 8 redes).

Cada red, cuesta aproximadamente de unos 2.500 a 3.000 sucres y dura únicamente una temporada, ya que se destruye pronto en contacto con maderas y otros cuerpos flotantes en el mar; así como también porque la misma jaiba destruye con sus pinzas las mallas de nylon. En suma, un equipo 
completo de pescar importa aproximadamente unos 20.000 sucres, cantidad que resulta muy difícil reunir.

Por lo antes expuesto, algunos pescadores para adquirir sus e- quipos han procedido a realizar un convenio con "El Chileno", el dueño de uno de los dos establecimientos turísticos de la playa. Según esto, el mencionado empresario financia las canoas y redes que adquieren los pescadores, y éstos liquidan poco a poco su deuda con la mitad de la pesca. La otra mitad también compra el mismo dueño del hotel, pero al precio del día. Este ingreso es el beneficio del pescador.

En el tiempo de la investigación, se pagaba hasta 25 sucres por un langostino, pero no pescaban más de 10 por cada redada, además de pequeños pescados y una que otra jaiba, que eran destinados al consumo doméstico.

En el tiempo no propicio para la pesca de camarones, los pequeños pescadores suelen alejarse hasta unos 1.000 metros de la costa para comprar las "pomadas" a los grandes pescadores de alta mar, para luego vender a los dueños de los hoteles, naturalmente la ganancia que obtienen en esta actividad es limitada.

En la propia temporada de pesca afirman que es posible obtener ganancias diarias hasta de 5.000 sucres; siendo lo mínimo que se alcanza unos 300 sucres que, en todo caso, es el doble de un jornal de agricultor.

Al concluir la época de pesca las ganancias fluctúan entre los 15.000 y 80.000 sucres por familia (de pescadores).

En todo caso cualquiera sea la ganancia de la pesca, no la invierten en mejorar o adquirir nuevo equipo de pesca; sino que la invierten en comprar material para la casa, en pagar las cuotas al IERAC; e inclusive en algunas ocasiones invierten en algún artículo de "lujo" razón por la cual cada temporada de pesca comienzan con un nuevo préstamo que se reembolsa de la manera ya expuesta.

\subsection{Actividades artesanales}

En la comunidad de Same no es posible constatar la existencia de una artesanía desarrollada, por no haber una tradición artesanal propia. En cambio, en Tonchigüe con su población más compleja y económicamente más diversificada, se desarrolla una amplia actividad artesanal. 
Las artesanías más frecuentes en Tonchigüe son la carpintería, albañilería, cerrajería, sastrería, zapatería, etc. Pero tampoco existe una artesanía más artística.

Entre los moradores de Same, sólo encontramos unas siete personas que son los denominados "ebanistas", comúnmente carpinteros, pero todos desarrollan otras actividades más, dedicándose a la carpintería únicamente cuando hay pedidos locales.

Un anciano recién llegado de Galera hace las atarrayas para la pesca en los esteros. Para concluir su obra demora más o menos un mes, dedicando una o dos horas diarias a este trabajo; cobra 500 sucres por la mano de obra, porque el cliente debe entregar el material necesario.

Otro viejecito trabaja esporádicamente como peluquero y un joven fabrica jaulas de bambú y como actividad recreativa construye modelos de buques pesqueros y otras naves, utilizando el palo de balsa. Estas artesanías no tienen un fin comercial, generalmente las regalan a los amigos. En cambio artesanías de este "mismo tipo, naturalmente algo mejor elaboradas, se venden en los mercados turísticos de Sua y Atacames, hasta por 2.000 sucres.

Un nuevo tipo de artesanía que está apareciendo, sobre todo entre las mujeres, es la elaboración de collares y brazaletes a base de discos pequeños de conchas y caracoles enlazados con hilo de nylon. Para la elaboración es preciso emplear mucho tiempo en la recolección de estas piezas en la arena de la playa.

Estos collares o brazaletes así elaborados resultan muy atractivos para los turistas, pero las personas que los fabrican entregan directamente a una señora de Quito, quien lleva a los almacenes folklóricos de la Capital.

Una mujer que se dedica a esta artesanía afirma que gana de 1.000 a 2.000 sucres en su tiempo libre, mientras cuida el palmar de un propietario que no vive en el lugar y que le paga 3.000 sucres mensuales por su labor de cuidado. 


\subsection{Otras ocupaciones}

Ya hemos anotado que a excepción de la agricultura, las demás actividades son escasas; hay muy poca ganadería y la pesca es únicamente por temporadas. Por tales motivos la forma más divulgada y base económica predominante en el lugar es el trabajo de jornalero en la agricultura.

El machete y el hacha son compañeros inseparables del jornalero que trabaja en los desmontes de las fincas cercanas al caserío.

Entre los jornaleros se encuentran tanto los que no tienen tierra propia y viven exclusivamente de este trabajo, como también aquellos pequeños propietarios, que además de cultivar sus solares, se dedican a trabajar para otros a cambio del salario establecido.

La rápida recuperación de la naturaleza favorecida por el clima tropical, es la mejor garantía para asegurarse un trabajo más o menos estable. El salario por el día de trabajo varía entre los 150 y 200 sucres y la persona que vive de esta actividad debe planificar sus gastos de acuerdo con los ingresos semanales de 800 a 1.000 sucres.

En busca de un trabajo mejor remunerado, particularmente los jóvenes, salen de su comunidad con destino a Esmeraldas u otras ciudades cercanas, en donde buscan empleo en diferentes trabajos, principalmente en fábricas. Otros jóvenes trabajan en talleres de diferente clase y aprenden algunas artesanías.

En cuanto a las mujeres jóvenes que todavía no se han juntado en unión libre con ningún hombre, salen muchas de éstas de su comunidad u optan por el trabajo doméstico en las grandes ciudades, incluyendo Quito y Guayaquil, que se encuentran relativamente distantes.

A veces, y en casos de familias de recursos económicos sumamente escasos, prestan la mano de obra de su hijo o hija todavía menor de edad; inclusive niños de cortos años y en plena edad escolar han sido separados de sus familias y enviados a las ciudades a prestar sus servicios domésticos. Tal fue el caso de una niña de 6 años que, según informan, fue enviada a trabajar con una familia de Guayaquil de donde desapareció al poco tiempo, sin que se haya vuelto a saber más de su destino. 
En comparación con Sua y Atacames, en el pequeño recinto de Same todavía no existe una verdadera actividad turística. Los dos hoteles instalados en la playa tienen una capacidad muy limitada. Tampoco la parroquia de Tonchigüe, dispone de buenas instalaciones turísticas, los pocos hoteles y salones tienen presentación muy sencilla y de escasa capacidad.

Por otra parte, se debe mencionar que tanto los moradores del recinto como los de la parroquia de Tonchigüe, no disponen de los medios necesarios para emprender en buenas instalaciones hoteleras, por lo cual la única alternativa que les queda es vender su mano de obra a los empresarios que ya están en posesión de los mejores sitios en la playa y que pronto comenzarán las construcciones.

Con una mayor afluencia turística, al menos los habitantes de estos pequeños caseríos podrán incrementar su pequeño negocio independiente: cigarrillos, bebidas, bocadillos, cocadas y las chucherías típicas del lugar.

Por lo demás, se debe reconocer que las playas de Same aún conservan su aspecto natural, limpio y tranquilo. Este estado semiparadisíaco, se perderá con el incremento del turismo, como ha ocurrido por ejemplo con las playas de Sua y Atacames.

\section{6. "El Grupo de Trabajo", la precooperativa}

Doce agricultores del recinto se han organizado en una especie de precooperativa denominada "El Grupo de Trabajo". Sus objetivos se centran en la actividad agrícola.

Se trata de una organización abierta a la participación voluntaria de los que desearen ingresar. Se prestan mutua colaboración en la preparación de la tierra (desmonte), la siembra, la cosecha y comercialización de los productos. Además facilitan el terreno para el cultivo a los socios que no disponen de él y se preocupan por la adquisición de los títulos de propiedad de los terrenos ocupados sólo por derecho de posesión.

Anteriormente existió otra precooperativa, que trató de obtener los títulos de propiedad sobre unas 500 hectáreas ocupadas, de un total de 10 o 15 mil hectáreas reclamadas por los herederos de u- no de los propietarios más grandes de la zona. Pero la negligencia de los socios, hizo que se quedaran en la mitad del camino, conformándose únicamente con promesas y sin la definitiva solución jurídica; por lo cual en la actualidad, los herederos 
de esa inmensa cantidad de terrenos, nuevamente amenazan con echarlos de las tierras, a no ser que paguen la cantidad de 2.000 por cada hectárea.

\subsection{Ferias, mercados y comercialización}

En el recinto de Same no se realiza una feria semanal. Los moradores acuden en parte a los mercados de Tonchigüe, que también son muy limitados y otros prefieren vender sus productos a los comerciantes intermediarios que pasan por el pueblo en las temporadas de cosechas o de pesca.

Esta forma de comercialización se ha visto favorecida por la nueva carretera que une estos lugares con las grandes ciudades.

De los compradores, algunos trabajan por su cuenta y otros lo hacen por encargo de ciertas empresas que dan tratamiento ulterior a los productos naturales.

Los precios son manipulados por los compradores, quienes previamente entran en acuerdos entre sí. Los agricultores son conscientes de esto, pero afirman que no les queda otra alternativa, ante la falta de un mercado regional que pueda recibir todos sus productos y, peor aún, ante la imposibilidad de contar con un medio de transporte propio para movilizarse con destino a las grandes ciudades.

De este modo se declaran más o menos satisfechos con las condiciones mercantiles reinantes.

En el mismo recinto viven dos personas que, por temporadas, se dedican a la actividad de intermediarios. Uno de ellos se ocupa de los productos agrícolas; y el otro, de nacionalidad chilena, es dueño de un hotel en la playa y se dedica a la comercialización de camarones y langostinos redados por los pocos pescadores de Same (véase: pesca).

\subsection{Necesidades, fondos disponibles y resumen de la situación económica}

El trabajo a jornal, es el único que permite una ocupación permanente e ingreso estable; las demás actividades son ocasionales, razón por la cual se hace imposible realizar un cálculo preciso de los ingresos de un trabajador en un determinado tiempo. 
En todo caso, la satisfacción de las necesidades está de acuerdo a lo que ganan. Esta "principio" se aplica tanto en la alimentación diaria como también otras necesidades de primer orden.

Se ha señalado ya que un jornalero gana entre los 150 y 200 sucres diarios y con estos ingresos tiene que afrontar los gastos de él y su familia.

Por lo general existen muy pocas posibilidades de un trabajo adicional; por ejemplo, la pesca de camarones es únicamente en una temporada y los trabajos adicionales para los carpinteros también son relativamente muy escasos.

En todo caso estas actividades proporcionan una ganancia irregular; ingresos que son gastados casi de inmediato en cualquier necesidad pendiente.

El agricultor y el ganadero, normalmente obtienen un superávit por la venta de sus productos o del ganado, que lo gasta a lo largo del tiempo que dura entre una y otra cosecha, o hasta la venta de una nueva res.

En muy raras ocasiones se invierte parte de este superávit en el mejoramiento de la agricultura o ganadería; por lo general no se nota el progreso en estas actividades económicas, justamente por la falta de inversión, reduciéndose todo a una economía de subsistencia.

En la mayoría de los casos los excedentes se emplean en la adquisición de materiales para la construcción o la ampliación de la casa, en la compra de ropa y uno que otro "artículo de lujo".

En el caso de quienes han utilizado prestamos para mejorar la agricultura o ganadería (que son muy pocos) con la venta de sus cosechas o del ganado, en primer lugar cancelan esos préstamos y, por lo general, les queda un excedente que es empleado en otras necesidades; este hecho puede ser considerado ya como una primera señal de cambio hacia una economía que supera, poco a poco, a la mera subsistencia.

Con respecto a los denominados "artículos de lujo", éstos comprenden: televisor, equipo de sonido, refrigeradora, etc., que por lo general son comprados a plazos. Se debe anotar además, que para el jornalero constituye un lujo, consumir de vez en cuando café instantáneo, enlatados o bebidas como la cerveza. 
Con excepción de los agricultores propietarios, de los pescadores con canoa y redes propias y de los ganaderos, los ingresos mensuales de una familia con un solo sueldo o jornal, apenas alcanza a los 4.000 sucres, suma que se agota en la adquisición de artículos de primera necesidad, en la compra de alguna ropa, etc., sin hablar siquiera de la necesidad de un tratamiento médico para alguno de la familia.

Los ingresos aumentan cuando uno $\mathrm{u}$ otro miembro de la familia realiza un trabajo ocasional, como los que hemos señalado anteriormente. En caso contrario se escucha en todas partes el mismo clamor: "no alcanza".

Sin embargo, las iniciativas por mejorar la situación económica son muy escasas; la gente ha optado por el conformismo y hasta causa la impresión de que aceptan esa situación con alegría.

\section{(8) APÉNDICE}

\subsection{Literatura selecta, (bibliografía)}

- Jaramillo M. Diagnóstico Socio-económico de la Provincia de Esmeraldas. Colección Pendoneros. 1981. Nro. 39, 10 A.

- 0.E.A. Los Déficits Educativos en América Latina. 1979.

- García Barrio C. Blacks in Ecuadorian Literature. En Whitten (ed.). Cultural Transformations and Ethnicity in Modern Ecuador. Chicago, 1981.

- I.N.E.C. IV Censo de Población y II de Vivienda, 1982; Resumen Provisional.

- Chávez A. Antología de Cuentos Esmeraldeños. Casa de la Cultura Ecuatoriana. Quito, 1960. 